Pedagogía y Saberes No. 50 Universidad Pedagógica Nacional

Facultad de Educación. 2019. pp. 199-210

\title{
La investigación educativa en contexto en Escuelas Normales Superiores
}

\section{Artículo de investigación}

\section{Educational Research in Context in Normal Schools \\ A pesquisa educasional em contexto em Escolas Normais Superiores}

Luz Mary Lache Rodríguez* Martha Cecilia Cedeño Pérez** Camilo Andrés Valderrama Alarcón***

\section{Para citar este artículo:}

Lache, L., Cedeño, M. y Valderrama, C. (2019). La investigación educativa en contexto en Escuelas Normales Superiores. Pedagogía y Saberes, 50, 199-210.

* Profesora del Departamento de Posgrado de la Universidad Pedagógica Nacional. Magíster en Educación e investigadora del Grupo Educación y regionalización en CTeI - GIER.

Correo electrónico: Imlacher@pedagogica.edu.co

Código ORCID: orcid.org/0000-0001-5637-7710

** Profesora del Departamento de Tecnología de la Universidad Pedagógica Nacional. Doctora en Antropología Social y Cultural e investigadora del Grupo Educación y regionalización en CTeI - GIER.

Correo electrónico: mccedenop@pedagogica.edu.co Código oRCID: orcid.org/0000-0002-3597-2876

*** Profesor del Departamento de Tecnología de la Universidad Pedagógica Nacional. Magíster en Desarrollo Educativo y Social e investigador del Grupo Educación y regionalización en CTeI - GIER.

Correo electrónico: cvalderrama@pedagogica.edu.co Código ORCID: orcid.org/0000-0002-5707-9675 


\title{
Resumen
}

Este artículo presenta los resultados de la investigación Producción y gestión de conocimiento en la investigación educativa en contexto en las Escuelas Normales Superiores (ENS) de Cundinamarca, que tuvo como objetivos identificar y clasificar las investigaciones adelantadas en los programas de formación de docentes durante el periodo 2010-2015, determinar las estrategias para realizar dichas investigaciones e identificar sus mecanismos de gestión y divulgación del conocimiento. La investigación trabajó desde un enfoque cualitativo con metodologías mixtas lo cual permitió establecer que la investigación es parte fundamental en el proceso de formación de maestros en ENS y que están articuladas a problemáticas contextuales aunque existen falencias en la visibilización del conocimiento que producen.

\section{Palabras clave}

formación docente; escuela normal superior; investigación formativa; gestión del conocimiento; apropiación social de conocimiento

\begin{abstract}
This article presents the results of the research titled Producción y gestión de conocimiento en la investigación educativa en contexto en las Escuelas Normales Superiores (ENS) de Cundinamarca, which aimed to identify and classify the research carried out in teacher training programs between the years 2010 and 2015, determine the strategies to conduct such investigations, and identify their knowledge management and dissemination mechanisms. The research worked from a qualitative approach with mixed methodologies, which made it possible to establish that research is a fundamental part in the process of teacher training in ENS and that they are linked to contextual problems, although there are flaws in the visibility of the knowledge they produce.
\end{abstract}

\section{Keywords}

teacher training; normal school; formative research; knowledge management; social appropriation of knowledge

\section{Resumo}

Este artigo apresenta os resultados da pesquisa Produção e gestão do conhecimento em pesquisa educacional no contexto das Escolas Normais Superiores (ENS) de Cundinamarca, que visou identificar e classificar as pesquisas realizadas nos programas de formação de professores no período 2010-2015, determinando as estratégias para realizar tais investigações e identificando seus mecanismos de gestão e disseminação do conhecimento. A pesquisa partiu de uma abordagem qualitativa com metodologias mistas que permitiu estabelecer que a investigação é parte fundamental no processo de formação de professores em ENS e que são articulados com problemas contextuais, embora existam falhas na visibilidade do conhecimento que produzem.

\section{Palavras-chave}

formação de professores; Escola Normal Superior; pesquisa formativa; gestão do conhecimento; apropriação social do conhecimento 


\section{Introducción}

工 as Escuelas Normales Superiores (ENS) en Colombia desempeñan un papel destacado en la formación inicial de maestros. Su existencia se hizo fuerte a partir de las primeras décadas del siglo $\mathrm{xx}$ y ha permitido tanto el acceso a la educación de diversas poblaciones en zonas rurales del país, como la preparación de personas en la docencia, de manera integral. De ese modo se configuran en instituciones formadoras de maestros y maestras en dos ámbitos básicos para la vida y los aprendizajes: la educación preescolar y la básica primaria.

En las ENS los procesos de formación en investigación son un eje esencial que se articula con un saber pedagógico y el conocimiento de sus regiones. En ese sentido, en las distintas ENS se adelantan procesos investigativos que se consolidan en trabajos de grado, particularmente en el programa de formación complementaria ( $\mathrm{PFC}$ ). Así, los procesos de investigación se constituyen en bastiones fundamentales que conectan los saberes de los maestros en formación con las problemáticas del entorno.

No obstante, hasta el momento son pocas las indagaciones sobre la situación formativa en investigación de los maestros en formación en las ENS en el departamento de Cundinamarca y en Colombia en general. Es decir, se desconoce gran parte de los procesos que se llevan a cabo, en la esfera de la producción de la investigación educativa en contexto y la apropiación social en los respectivos entornos, aspectos esenciales a la hora de trazar políticas públicas en relación con la formación de maestros.

En este artículo se presentan los resultados de la investigación cuyo propósito fundamental fue dilucidar la producción investigativa en contexto y la gestión de conocimiento realizada en el PFC de cinco ENS del departamento de Cundinamarca (Colombia) entre los años 2010 y 2015. Por ello, en primer término, se esbozan algunos elementos teóricos básicos que sustentaron y dieron sentido al proceso investigativo; en segundo lugar se determinan los presupuestos metodológicos fundamentales, y, por último, se presentan los resultados y las conclusiones.

\section{Elementos teóricos básicos}

La investigación es entendida como un referente de calidad en dos dimensiones. La primera, relacionada con la formación inicial, en tanto que la formación en investigación crea las condiciones para que los estudiantes hagan una reflexión crítica sobre su propio ser y quehacer, para que aprendan a observar, analizar y entender lo que hacen desde su práctica pedagógica, permitiendo la configuración de una cultura de la investigación. La segunda dimensión está relacionada con la institución, mediante el desarrollo de líneas y proyectos de investigación que les permitan comprender su organización y el rol de sus actores, problematizar su propuesta de formación, desarrollar alternativas pedagógicas y realizar los procesos evaluativos necesarios para garantizar la calidad de sus servicios (MEN, 2000, p. 31).

De esta manera, la investigación se entiende como la indagación permanente de las prácticas de aula, que incluye las discusiones de aspectos como las didácticas, los enfoques pedagógicos, las formas de aprender y de enseñar, las comunidades educativas, las reconfiguraciones curriculares, entre otras. Así, la investigación educativa está asociada a la sistematización permanente de las acciones pedagógicas que permiten la delimitación de lo que se hace y de cómo se hace, en función de generar saberes para un contexto. En este sentido, "la investigación también intenta constituir saber universal por la relación que establece con políticas educativas nacionales o internacionales, o simplemente con tendencias emergentes para la reflexión educativa" (MEN, 2015, p. 26).

Para el Ministerio de Educación (MEN), la investigación es uno de los factores fundamentales para la acreditación de alta calidad de los PFC de las ENS, tal como se refleja en el Decreto 4790 de 2008, por el cual se establecen las condiciones básicas de calidad y se dictan otras disposiciones. En el artículo 3 de dicho documento titulado Condiciones básicas de calidad, se esboza la importancia de las "innovaciones en el campo educativo que fomenten el desarrollo del pensamiento crítico investigativo". Ello también se evidencia en el documento marco de acreditación de calidad y desarrollo de las ENS (2000) en el capítulo "Referentes y criterios de calidad en la Escuela Normal Superior", en el cual considera la investigación educativa como

un referente de calidad dado que contribuye a la creación de una cultura de la investigación en las Escuelas Normales Superiores y a la generación del espíritu investigativo en los maestros en formación, como punto de partida para fomentar la curiosidad y el deseo de conocer la realidad educativa de forma racional y objetiva. (MEN, 2000, p. 30).

Por su parte, el Consejo Nacional de Acreditación de Escuelas Normales Superiores (CAENS), en un documento del año 2000 denominado Formación de maestros, elementos para el debate, coincide con las propuestas planteadas desde el MEN y Asociación Nacional de Escuelas Normales (ASONEN), al conside- 
rar que la investigación se configura en un elemento que transversaliza tanto el quehacer institucional como las propuestas de formación de las ENS y, por ende, se convierte en un factor estratégico desde el cual puede gestionarse el conocimiento. Por tanto, no bastaría con asumir algunas asignaturas en el plan de estudios que tengan que ver con la investigación, sino organizar y desarrollar tanto una actitud de indagación, como una experiencia investigativa de los maestros, de los estudiantes y de las instituciones para agenciar la comprensión del mundo, la posibilidad de cambio y la calidad de la acción educativa institucional, tal como plantean Calvo, Camargo y Pineda (2008) al afirmar que

\begin{abstract}
la importancia y valor de la investigación se releva en cuanto producción de conocimiento, no sólo como medio para orientar la acción educativa y pedagógica, a partir de los hallazgos y el acumulado de saber producido, sino como valor en sí misma, por ser el conocimiento patrimonio de la cultura y por ser, en sí, cultura [...] Este conocimiento plantea, al individuo, un lugar de comprensión del mundo, una apertura del campo de posibilidades para su acción y una inteligibilidad de sus relaciones, entre otras perspectivas. (p. 166).
\end{abstract}

Lo anterior significa que la investigación comprende la reflexión de los maestros sobre su quehacer; al revisarlo, lo interrogan y producen un saber y hacer específico del trabajo en la escuela (qué se enseña, cómo, a quién, con qué medios, orientaciones y en qué contextos), en tanto la pedagogía es un saber extremamente anclado en la práctica. En tal sentido

\begin{abstract}
los maestros se constituyen en analistas de su quehacer, en autoobservadores de lo que sucede en sus aulas de clase, en investigadores de los objetos de conocimiento disciplinares que ponen en juego pedagógicamente y en profesionales que aportan saber y conocimiento a la pedagogía. La investigación pedagógica se constituye en un medio para la inserción del maestro en la toma de conciencia de sus prácticas y en las gramáticas que subyacen a los saberes y en su forma de circulación. (Martínez, 1999, citada por Calvo, Camargo y Pineda, 2006, pp. 166-167).
\end{abstract}

En la formación de maestros y maestras es relevante que se comprenda la docencia como una actividad profesional que está en la base de una práctica y un discurso que la legitiman y que todos los elementos desarrollados en la investigación deben subyacer a la intención de producción de saber pedagógico, pues tal como lo enuncia Herrera (2010) en la formación investigativa del profesorado se debe apuntar a que los recursos teóricos, la reflexión epistemológica y metodológica, los procedimientos y las técnicas se enfoquen hacia la explicitación del saber pedagógico y no hacia la producción de conocimientos con una pretensión de generalidad de los procesos educativos. (p. 62).

Lo anterior remitirá siempre a la necesidad de precisar el tipo de investigación en la que los maestros y maestras deben ser formados en aras de contribuir a la transformación de las prácticas pedagógicas.

\section{Gestión del conocimiento en la investigación educativa en contexto}

En lo que respecta al concepto de gestión del conocimiento se acogió la definición de Nonaka et al. (1999, citados en Farfán y Garzón, 2006) que lo postula como

\begin{abstract}
un sistema facilitador de la búsqueda, codificación, sistematización y difusión de las experiencias individuales y colectivas del talento humano de la organización, para convertirlas en conocimiento globalizado, de común entendimiento y útil en la realización de todas las actividades de la misma, en la medida que permita generar ventajas sustentables y competitivas en un entorno dinámico [...] una secuencia ascendente de los datos (hechos objetivos de acontecimientos) generando información (mensaje: emisor/receptor) que requiere de la clasificación y codificación para convertirse en conocimiento que por medio de la internalización promueve la creación de espacios propicios para la aplicación de ese conocimiento, llevándolo a la acción y generando nuevamente datos e información para la creación de nuevos conocimientos. (p. 8).
\end{abstract}

Así, la gestión del conocimiento en la investigación educativa en contexto significa que cada persona que tiene que ver con ella, le agrega valor al filtrarla, al sintetizarla, al interpretarla y depurarla, en términos de una orientación hacia el conocimiento pedagógico que lo genere, lo facilite, lo alimente, lo difunda y lo transforme. Esto es, intentar entender qué hace el conocimiento, de dónde viene, cómo se crea y cómo contribuye a procesos continuos y continuados de aprendizaje y desaprendizaje, tanto de los sujetos como de la ENS. En tal sentido el concepto de gestión de conocimiento tiene varias dimensiones, a saber: a) el proceso de producción del conocimiento por medio de los aprendizajes organizacionales ${ }^{1}$; b) el espacio

1 Los conocimientos organizacionales derivan de las experiencias o conocimiento tácito de su personal, el cual se sintetiza e incorpora en las actividades claves de la organización para 
de conocimiento (región, ciudad, organización) o lo que se denominaría contexto ${ }^{2}$; c) las herramientas y tecnologías de gestión del conocimiento que guardan y documentan el conocimiento organizacional; d) la sinergia como dinámica del proceso de desarrollo de un sistema; e) la capacidad de respuestas de las comunidades y los individuos frente a nuevos problemas o desafíos en un medio inestable y cambiante; y f) los trabajadores del conocimiento (en este caso, los maestros en formación y los maestros y directivos de la ENS).

La gestión del conocimiento se comprendió como una manera de organizar, sistematizar, almacenar, distribuir, socializar y poner a disposición de la ENS, sus integrantes y sus comunidades, su propio conocimiento, en este caso de la investigación en contexto que allí se realiza; esto es, conocer lo que se sabe, tanto desde lo implícito como desde lo explícito, evitando los monopolios de información. Tampoco implica que se base en la eficiencia y productividad de las ENS pero sí en contar con una memoria activa de la investigación que permita tener estados de la cuestión y proyecciones en relación con esta y la formación en investigación, aunque dicho conocimiento se haya producido en diferentes contextos y por diferentes sujetos. Y, en tal sentido, la creación de nuevo conocimiento es producto de una interacción dinámica entre sus productores, esto es, la interacción que puede darse en comunidades de práctica, en donde maestros, estudiantes y directivos se dispongan a la revisión crítica de lo que se hace y se tiene en la ENS.

Por tanto, en la perspectiva de la producción y gestión de conocimiento en la investigación en contexto de las ENS se requiere generar condiciones que permitan a los maestros de la ENs hacer explícito su conocimiento tácito, así como espacios de encuentro, socialización y trabajo colectivo, que permitan que dichos conocimientos se socialicen y puedan integrarse en el conocimiento colectivo de la ENS. De igual manera, establecer un conocimiento de base sobre el contexto de cada ENS en el que ejerce su actividad, como institución y a partir de los sujetos que la componen, que permita evidenciar cómo impacta dicha actividad en las comunidades que participan en las

impulsar el proceso continuo de innovación (Farfán y Garzón, 2006, p. 9).

2 Como contexto no solamente se hace referencia al contexto de desarrollo de las prácticas pedagógicas de los estudiantes en formación, sino al contexto mismo de la ENS, dado que, a pesar de compartir ciertas características, cada ENS es distinta. investigaciones que se desarrollan, bien sea desde la práctica formativa o desde la investigación en cabeza de los formadores o de carácter institucional.

\section{Diseño metodológico}

La investigación que se llevó a cabo tuvo un enfoque cualitativo que permitió un acercamiento a la realidad en estudio, cinco Escuelas Normales Superiores del departamento de Cundinamarca (Colombia), de una manera flexible para dar cuenta de sus contornos, aristas y movimientos. Así, fue posible una aproximación interpretativa y naturalista a los fenómenos de interés para darles sentido teniendo en cuenta los significados que las personas les otorgan, tal como lo enuncian Denzin y Lincoln (2005).

Este enfoque condujo a la recolección de material empírico a través de diversas estrategias como la revisión documental de trabajos de grado de los estudiantes del PFC y de manuales de investigación o publicaciones de las ENS, el desarrollo de entrevistas con rectores y profesores del programa y encuestas con los estudiantes del mismo. También, se hizo un rastreo en internet de los trabajos de investigación publicados en aras de evidenciar procesos de divulgación que vayan más allá de los espacios institucionales.

Desde la revisión documental se logró esclarecer el tipo de investigación que se lleva a cabo en cada ENS, las líneas y las principales temáticas abordadas; al tiempo que hizo posible conocer las formas en las cuales el conocimiento producto del proceso investigativo es visibilizado dentro del ámbito escolar y local.

En cuanto a la entrevista semiestructurada se dirigió especialmente a los rectores y rectoras, y al cuerpo docente que tiene la responsabilidad de liderar la investigación en las instituciones. Se realizaron dos entrevistas en La Palma a maestros formadores del PFC; en Quetame se entrevistó al rector y al docente encargado del área de investigación del PFC; en Pasca una entrevista colectiva en la que estuvieron presentes la rectora, la coordinadora de investigación y la coordinadora del PFC; en Junín se entrevistó a la rectora y a dos profesoras del PFC que tienen a cargo la formación en investigación, y en Ubaté se hizo una entrevista al rector y otra a un profesor del PFC.

En lo que respecta a las encuestas se debe mencionar que se aplicaron 70 a estudiantes del PFC de la siguiente manera: 12 en Quetame, 19 en Ubaté, 11 en Junín y 28 en Pasca. En la ENS de La Palma no se pudo aplicar ninguna debido a dinámicas propias de la 
institución que hicieron imposible llevar a cabo esta actividad con el estudiantado del PFC. No obstante lo anterior, la aplicación de dicha encuesta en las otras ENS brindó una información relevante a la hora de determinar las condiciones en las cuales se realizan los procesos investigativos, la importancia que estos tienen dentro de la formación docente y la manera como estos se insertan en la práctica docente.

El modelo de análisis seguido en la investigación fue el siguiente:

Tabla 1. Modelo de análisis de la investigación

\begin{tabular}{|c|c|c|c|}
\hline Categoría teórica & Categoría de análisis & Subcategorías de análisis & Metodología/Instrumento \\
\hline \multirow{7}{*}{$\begin{array}{l}\text { Producción de } \\
\text { conocimiento }\end{array}$} & \multirow{3}{*}{$\begin{array}{l}\text { Investigaciones adelantadas } \\
\text { en el PFC de las ENS durante } \\
\text { el periodo } 2010-2015 .\end{array}$} & Líneas de investigación. & \multirow{3}{*}{$\begin{array}{l}\text { Revisión documental. } \\
\text { Matriz de análisis de } \\
\text { investigaciones. }\end{array}$} \\
\hline & & Proyectos de investigación. & \\
\hline & & $\begin{array}{l}\text { Investigación de los } \\
\text { maestros formadores. }\end{array}$ & \\
\hline & \multirow{4}{*}{$\begin{array}{c}\text { Estrategias de las ENS para } \\
\text { desarrollar procesos inves- } \\
\text { tigativos en la formación } \\
\text { de los estudiantes del PFC. }\end{array}$} & $\begin{array}{c}\text { Naturaleza de los proyectos } \\
\text { de investigación. }\end{array}$ & \multirow{4}{*}{$\begin{array}{l}\text { Entrevistas semiestruc- } \\
\text { turadas con rectores y } \\
\text { profesores del PFC. } \\
\text { Encuestas a estu- } \\
\text { diantes del PFC. }\end{array}$} \\
\hline & & $\begin{array}{c}\text { Estrategias para la } \\
\text { formación en investigación. }\end{array}$ & \\
\hline & & $\begin{array}{l}\text { Gestión y financiación } \\
\text { de recursos. }\end{array}$ & \\
\hline & & $\begin{array}{c}\text { Articulación entre } \\
\text { investigación y práctica. }\end{array}$ & \\
\hline \multirow{2}{*}{ Gestión de conocimiento } & \multirow{2}{*}{$\begin{array}{c}\text { Estrategias empleadas por } \\
\text { las ENS para la apropiación } \\
\text { y divulgación del conoci- } \\
\text { miento generado a partir } \\
\text { de sus investigaciones. }\end{array}$} & $\begin{array}{l}\text { Estrategias para la } \\
\text { divulgación de la } \\
\text { investigación. }\end{array}$ & \multirow{2}{*}{$\begin{array}{l}\text { Revisión documental. } \\
\text { Entrevistas semiestruc- } \\
\text { turadas con rectores y } \\
\text { profesores del PFC. } \\
\text { Encuestas a estu- } \\
\text { diantes del PFC. }\end{array}$} \\
\hline & & $\begin{array}{l}\text { Formas de apropiación } \\
\text { social de la investigación. }\end{array}$ & \\
\hline
\end{tabular}

Fuente: elaboración propia.

\section{Resultados}

En lo que respecta a la producción de conocimiento, en términos de las investigaciones adelantadas en el PFC de las ENS durante el periodo de estudio (20102015), la revisión documental permitió determinar que la mayoría de ellas son trabajos de grado (144), aunque también se pudo encontrar algunos artículos breves publicados en la revista Albores Pedagógicos Teresianos de la Ens de Quetame (12) e informes finales de investigación del Programa Ondas ${ }^{3}(10)$. Cabe destacar que no se encontraron documentos de este tipo publicados en línea ni en otras revistas locales, regionales o nacionales.

3 El Programa Ondas es la estrategia fundamental del Departamento Administrativo de Ciencia, Tecnología e Innovación (Colciencias) en Colombia para fomentar la cultura ciudadana en CTeI en niños, niñas y jóvenes de Colombia.
En lo que respecta a la distribución anual de esas publicaciones se tiene que entre 2010 y 2015 la mayor productividad en cuanto a los trabajos de grado está en las ENS de Ubaté (55) y Pasca (52), siendo más reducida las de La Palma (17), Junín (12) y Quetame (8). Paradójicamente, es en esta última institución en donde ha habido mayor socialización de los procesos investigativos al ser visibilizados parte de sus resultados en la revista ya mencionada. Lo anterior significa que, en términos generales, los trabajos de investigación y la forma como estos se presentan corresponden a tres modalidades a saber: trabajos de grado, artículos breves en revistas e informes de proyectos Ondas.

En cuanto a las problemáticas abordadas y la metodología empleada, en los trabajos prevalecen temáticas relacionadas con problemas educativos, sociales y ambientales, tal como se muestra en la tabla 2. En lo que respecta a la metodología y técnicas 
utilizadas para la recolección de datos se encontró que están ligadas al enfoque cualitativo, cuantitativo y mixto; por tanto, se han utilizado técnicas relacionadas con dichos marcos tales como la revisión documental, la entrevista, la observación y la encuesta, entre otras. Aquí vale la pena mencionar que en la mayoría de los trabajos revisados se observa una cierta claridad en el esbozo de la propuesta en lo que concierne a la definición de la problemática, la metodología y los referentes teóricos, aunque en algunos casos se aprecie también precariedad en este último aspecto.

Tabla 2. Áreas temáticas relevantes de los trabajos de grado

\begin{tabular}{|c|c|c|}
\hline Nombre de la ENS & $\begin{array}{c}\text { Número de trabajos de } \\
\text { grado 2010-2015 }\end{array}$ & Temáticas relevantes abordadas \\
\hline $\begin{array}{l}\text { ENS Nuestra Señora de la } \\
\text { Encarnación de Pasca }\end{array}$ & 52 & $\begin{array}{l}\text { Pensamiento científico y matemático. } \\
\text { Enseñanza de las matemáticas y las ciencias naturales. } \\
\text { Competencias lectoescritoras. } \\
\text { Medio ambiente. }\end{array}$ \\
\hline $\begin{array}{l}\text { ENS Santa Teresita } \\
\text { de Quetame }\end{array}$ & 8 & $\begin{array}{l}\text { Las TIC como mediaciones pedagógicas. } \\
\text { Las inteligencias múltiples. } \\
\text { El juego como estrategia didáctica. } \\
\text { Educación ambiental. } \\
\text { Pensamiento matemático. }\end{array}$ \\
\hline ENS de Junín & 12 & $\begin{array}{l}\text { Medioambiente. } \\
\text { Pedagogía y didáctica. } \\
\text { La lectoescritura. } \\
\text { Las Tic. } \\
\text { Competencias comunicativas. }\end{array}$ \\
\hline ENS de Ubaté & 55 & $\begin{array}{c}\text { Pedagogía y didáctica. } \\
\text { Habilidades comunicativas. } \\
\text { Medioambiente. } \\
\text { Trabajo colaborativo. } \\
\text { Los derechos de los niños y las niñas. } \\
\text { La lectoescritura. } \\
\text { Educación de adultos. }\end{array}$ \\
\hline $\begin{array}{c}\text { ENS La Divina Providencia } \\
\text { de La Palma }\end{array}$ & 17 & $\begin{array}{l}\text { Competencias comunicativas. } \\
\text { La lectoescritura. } \\
\text { Competencias cívicas. } \\
\text { Medioambiente. } \\
\text { Vocación docente. }\end{array}$ \\
\hline
\end{tabular}

Fuente: elaboración propia.

Con respecto a los énfasis de las investigaciones en las ENS, una primera mirada a los distintos trabajos encontrados permite observar algunos elementos de cierta relevancia. El primero tiene que ver con la formalización de los procesos investigativos y su dilucidación, esto es, con la manera en que son formulados y correctamente sustentados en referentes teóricos y metodológicos básicos. En ese sentido, se observan dificultades en el esbozo de las referencias teóricas, lo cual hace que se aprecie cierta precariedad y falta de rigor en ellas. El segundo tiene que ver con lo metodológico que en ciertos trabajos no se esboza de manera explícita, por tanto, no es claro el enfoque de investigación, las técnicas y el análisis de datos. Desde ese punto de vista se acude entonces a una mirada casi intuitiva sobre el problema que se quiere abordar. A nivel formal, también cabe mencionar los problemas de redacción e incluso de ortografía que se observan en la mayoría de los trabajos revisados.

Así que, una vez hecho el análisis, se identifican algunos énfasis relevantes que se agrupan en las siguientes categorías: pedagogía y didáctica, ambiental, pensamiento científico y matemático, competencias comunicativas y lectoescritoras, convivencia y ambiente escolar, y memoria y cultura. Cabe mencionar que esta taxonomía solo es una manera de 
identificar las áreas generales más trabajadas, lo cual no es óbice para que en algunas oportunidades, se encuentren y se entremezclen, y ello se evidencia en algunos trabajos interdisciplinarios que tocan varias temáticas. Por tanto, en ningún momento los énfasis descritos arriba son excluyentes sino por el contrario, conforman un todo que ha sido desglosado solo para poder describirlos, para pasarlos por el tamiz del análisis, con el ánimo de comprender la esencia de los procesos investigativos llevados a cabo en cada una de las ENS estudiadas.

En cuanto a la investigación que realizan los maestros formadores de las ENS, realmente se evidencia muy poco. Tanto en la revisión documental como en las entrevistas y las encuestas, es notorio que a pesar de que algunos maestros en la institución desarrollan proyectos de investigación, no hay la suficiente sistematización y divulgación en la ENS y, por ende, la visibilidad es baja.

Por lo regular, los proyectos que desarrollan los maestros son los que se postulan al Programa Ondas de Colciencias y la sistematización de estos atiende la exigencia que dentro de dicho programa se hace a partir de bitácoras, pero es recurrente que de estos informes no haya un registro en algunas de las ENS, aun cuando se sabe de su participación en dichos procesos. En otras ocasiones, se desarrollan proyectos transversales que se enfocan en la convivencia, la comunicación, la educación sexual o los Proyectos Ambientales Escolares (PRAE); allí se cuenta con la participación de toda la comunidad educativa y no solamente de quienes participan en el PFC.

Finalmente, puede indicarse que hay proyectos que los maestros formadores en las ENS vienen desarrollando desde su quehacer (proyectos de aula) y que posteriormente van obteniendo reconocimiento, tanto interno como externo y algunos de ellos pasan a ser instituidos dentro de la ENS. Sobre estos últimos podría mencionarse que se concentran en áreas como ciencias naturales y matemáticas, en el caso de Pasca; en convivencia para el caso de La Palma, y en inglés en Quetame.

Con respecto a la naturaleza de los proyectos de investigación y las estrategias de las ENS para desarrollar procesos investigativos en la formación de los estudiantes del PFC se logró identificar una ruta de desarrollo, más o menos común, de los procesos de investigación de los estudiantes, ligados a su práctica y que se presenta en la figura 1.

Esta ruta, básicamente, comprende cinco etapas, aunque sus desarrollos sean dispares en las ENS. La primera etapa corresponde a una fundamentación teórica sobre la investigación en términos de lo epistemológico y lo metodológico. En esta etapa puede indicarse que se hace énfasis en la diferenciación de los paradigmas y enfoques de investigación y se pretende que se diferencien etapas y procesos de la investigación.

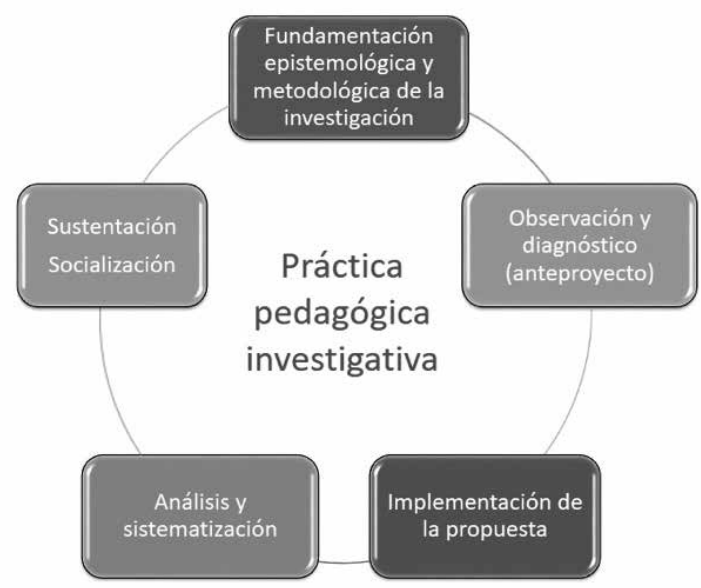

Figura 1. Ruta de desarrollo de los procesos de investigación de los maestros en formación en las ens de Cundinamarca

Fuente: elaboración propia.

La segunda etapa tiene que ver con la elaboración del anteproyecto, desde la observación y el diagnóstico de problemáticas que los estudiantes evidencian en sus prácticas pedagógicas. Aquí se realiza una lectura de contexto o cartografía y se elabora un diagnóstico a partir de entrevistas, encuestas, pruebas o test de conocimientos, historias de vida, que les permite plantear una pregunta problema y una caracterización de la población. En la tercera etapa se plantea la propuesta pedagógica o derivación práctica y se implementa en los diversos lugares de la práctica. En estas propuestas los estudiantes plantean lo que van a implementar para tratar de mejorar la situación problema identificada y, en tal sentido, planifican las actividades pedagógicas que van a desarrollar, así como los instrumentos mediante los cuales van a evaluar los resultados de la intervención.

La cuarta etapa se enfoca en la sistematización, análisis de la información y producción del documento final (en la mayoría de los casos, trabajos de grado, y con menor frecuencia, artículos académicos). En esta etapa también es fundamental la reflexión pedagógica que los estudiantes en formación desarrollan frente a la práctica investigativa, en donde establecen logros, dificultades y los cambios o impac- 
tos que el desarrollo de su propuesta ha realizado en las poblaciones con las que trabajaron; ; es decir, que se promueve una reflexión personal, pedagógica y social.

La quinta y última etapa es la sustentación y socialización de los trabajos finales de los estudiantes, aunque hay ENS en donde en segundo o tercer semestre se realiza la puesta en común de los avances de su proyecto a la fecha. En esta etapa hay diversidad de formas, que van desde la tradicional sustentación de los proyectos realizados ante un jurado compuesto por maestros formadores o por invitados de instituciones con las que se tienen convenios para que los estudiantes continúen su formación como licenciados, pasando por coloquios, seminarios o encuentros de investigación y que pueden o no, coincidir con eventos institucionales (como la semana normalista o feria) o eventos que convoca el municipio (programados por la Secretaría de Educación o de Cultura). Estos eventos de socialización y sustentación permiten visibilizar a la comunidad educativa o a la comunidad local (e incluso a otros actores del ámbito educativo y de la investigación) lo que los futuros maestros han desarrollado en el programa de formación complementaria en cada ENS.

Si bien es cierto que los proyectos de investigación parecieran, por lo general, emerger de la práctica y las necesidades del contexto, puede indicarse que no es la única vía. Los procesos de investigación de los estudiantes también proceden de su participación en proyectos de investigación que formulan los maestros formadores o en macroproyectos, en procesos que pueden ser cíclicos, lo que significa que se da continuidad desde el primer semestre a los proyectos que han tenido trascendencia y por los que ya ha transitado otra(s) cohorte(s) de estudiantes durante cuatro semestres.

Otra fuente de los proyectos de investigación la constituyen los trabajos de grado que han realizado los estudiantes de las anteriores cohortes, aunque dicho material en ocasiones no tenga una organización y disponibilidad en la ENS. Estos trabajos también son recursos mediante los cuales los estudiantes pueden establecer estados de la cuestión sobre sus propios problemas de investigación, pero no en todos los casos se tienen en cuenta. Esta es una medida en la que se considera que los trabajos de grado de los estudiantes y la producción de conocimiento en la ENS tiene una apropiación.

4 Vale la pena mencionar que el instrumento utilizado para tal fin es la Pedagotácora en la ENS de Quetame.
En el desarrollo de la práctica investigativa, una estrategia fundamental para la formación es el acompañamiento a los estudiantes. La práctica se desarrolla en las sedes de la ENS o en otras escuelas, generalmente del ámbito rural o también urbano, dependiendo del tipo de práctica y la cantidad de estudiantes que se tenga para desarrollarla. Para este acompañamiento se presenta, por lo general, la asesoría de los maestros formadores del PFC que tienen como responsabilidad orientar la investigación y, dependiendo del proyecto que se acompaña, hay un asesor en el campo disciplinar. También se considera al maestro cooperador o titular en el campo de práctica como un acompañante de estos procesos. Una herramienta que contribuye sustancialmente a este acompañamiento es el diario que el estudiante lleva durante su práctica en el cual los maestros formadores pueden hacer seguimiento a su proceso y complementar con las visitas que realizan para observar los desarrollos de la práctica.

Por otra parte, se evidenció que la gestión de los proyectos de investigación en el PFC se desarrolla a través de convenios de práctica y con el concurso de los maestros formadores desde su acompañamiento y también se utilizan recursos provenientes, por ejemplo, del programa Ondas de Colciencias o se aprovechan los recursos de los proyectos externos que llegan a la ENS. Sin embargo, se hacen esfuerzos para apoyar los proyectos de investigación, de manera institucional, con recursos propios que, generalmente, están representados en materiales de apoyo. En algunos casos, al ser los proyectos de investigación, proyectos productivos, se aprovechan sus ganancias (generadas de la venta de productos) para reinvertirlas en el mismo proyecto; en otros, se buscan los recursos con el municipio o los proveen los estudiantes para el caso de su práctica pedagógica investigativa.

El tiempo que se dedica en el PFC para la formación en investigación también es relevante. Por lo general, se cuenta con una hora semanal para la investigación, lo que al parecer en ciertos casos dificulta el desarrollo de los procesos investigativos de los estudiantes. En correlación, los tiempos con que cuentan los maestros formadores para orientar estos procesos no son suficientes (en algunos casos no hay asignación académica para ello y, en otros, se han pagado horas extras) por la cantidad de estudiantes que deben asesorar y ello, en la mayoría de los casos, evidencia la ausencia de un colectivo de profesores que se aglutinen en torno a la investigación ${ }^{5} \mathrm{y}$ a la

5 En la mayoría de las ENS se tiene la perspectiva de configurar grupos de investigación en donde al menos se encuentren 
formación en investigación y de una política en la que se dé relevancia al tiempo para la investigación y la formación en investigación.

En lo que respecta a la articulación entre práctica pedagógica e investigación se encontró que el 93\% del estudiantado reconoce dicho nexo a través de la realización de sus proyectos de práctica pedagógica; otra evidencia de ello es que el $81 \%$ identifica una o más líneas de investigación en la institución en que están definidas. Lo anterior permite pensar que efectivamente las ENS han consolidado una trayectoria investigativa teniendo como base fundamental la práctica pedagógica. Esta articulación también puede verse en la importancia que las ENS otorgan a la investigación, dada su naturaleza de formadoras de maestros. En ese sentido, el 99\% del estudiantado consideró que para la institución es importante la investigación en su formación y eso se comprueba en el hecho de que un $85 \%$ ha participado en proyectos de investigación que son asesorados por los docentes.

La socialización de los trabajos de indagación en las ENS se lleva a cabo a través de distintas estrategias. Una de ellas es la sustentación pública, las ferias o encuentros en los cuales se presentan dichos procesos. Aunque estas sustentaciones son una oportunidad para que la comunidad educativa conozca las experiencias investigativas, no se deja una memoria que evidencie la sistematización de estos eventos. No obstante, esta divulgación permite vincular a la sociedad con los objetivos de las investigaciones, ya que el conocimiento producido cumple una función social dentro de un contexto distinto a la comunidad que lo produce. Otra es la presentación de los trabajos de investigación tanto de estudiantes del PFC como de maestros formadores, en escenarios externos a la ENS tales como ferias municipales o programadas por la Secretaría de Educación u otras secretarías; en encuentros, seminarios, coloquios, nacionales e internacionales y otros espacios en los que los maestros formadores identifican que se pueden presentar los proyectos.

Se pueden mencionar también otras estrategias de socialización tales como la publicación de los trabajos de investigación en revistas, pues algunas ENS cuentan con una publicación en donde incluyen resúmenes de las investigaciones y proyectos de grado de los estudiantes ${ }^{6}$; no obstante, su gestión

adscritos los maestros formadores que orientan los procesos de indagación del estudiantado.

6 Como en Pasca (Semillas de Investigación) y Quetame (Albores Pedagógicos). se torna difícil por cuenta del tiempo y los recursos, por lo cual no hay una continuidad en su edición; ello hace imprescindible contar con un equipo que esté al frente de este proceso de manera regular. Otra estrategia es la publicación de trabajos de investigación en internet; sin embargo, se encontró que esta herramienta es poco utilizada por las instituciones, lo cual limita sustancialmente dar a conocer a la sociedad sus propuestas y desarrollos investigativos. Lo anterior significa que, en términos generales, las ENS no usan internet para socializar los distintos proyectos de investigación que adelantan, lo cual hace que estas propuestas sean poco conocidas en la sociedad. Otra característica de estos procesos de socialización es que no se hacen de manera institucionalizada, sino que son los estudiantes y/o maestros los que de forma individual dan a conocer las actividades académicas de las ENS.

En cuanto a las formas de apropiación social de la investigación cabe resaltar primero que ello hace referencia al logro de cuatro objetivos fundamentales a saber: crear almacenes de conocimiento tanto externo como interno; facilitar la transferencia de conocimiento entre individuos; construir un entorno que permita la creación, transferencia y uso del conocimiento de manera más eficiente; y lograr que la organización se concentre en mejorar los resultados organizativos. Desde estos referentes, las ENS tienen serias dificultades en los procesos de apropiación social del conocimiento pues se requiere de equipos de trabajo dedicados a los procesos de gestión de conocimiento y estas instituciones carecen de ello por varias razones. Una de ellas radica en que la normatividad vigente no permite la dedicación de maestros a estas actividades, y otra, en que los maestros no cuentan con la formación pertinente para desarrollar estos procesos. Una más se enfoca en la alta rotación de maestros y, por último, en las dificultades que existen en el almacenamiento de la información. Lo anterior trae como consecuencia que las experiencias de formación en investigación no cuenten con secuencias de trabajo claras y de manera frecuente se deben iniciar los procesos desde cero, generándose así un desgaste institucional. Los ejercicios investigativos, en la mayoría de los casos, se personalizan y no se institucionalizan, perdiéndose la experiencia acumulada en el momento en que el docente se retira o porque sencillamente no desea continuar trabajando en el tema. Ahora bien, en lo que atañe al conocimiento del estudiantado de los proyectos de investigación, en las encuestas realizadas se encontró que un $83 \%$ sabe de estos y un $73 \%$ tiene conocimiento de proyectos desarrollados por 
los docentes de la ENS; mientras que un 93\% corrobora que los ejercicios de investigación adelantados son socializados con la comunidad educativa.

En este análisis pueden registrarse algunas dinámicas fluctuantes que interfieren en los procesos de investigación en la formación de los normalistas. Estas dinámicas tienen que ver, por un lado, con la asignación y permanencia del profesorado para el acompañamiento de los procesos de investigación y para el desarrollo de sus propios procesos de investigación, pues en algunas ENS el cuerpo docente que orienta la investigación tiene vinculación provisional, lo que lo convierte en una población flotante que se lleva los saberes y procesos cuando se desvincula de la institución. Lo anterior, unido a la ausencia de conocimiento colectivo de lo que se desarrolla en las ENS, produce procesos intermitentes que no agencian la constitución de colectivos de investigación permanentes.

Hay otros factores importantes que también inciden en la situación descrita hasta ahora como, por ejemplo, que es necesario establecer un perfil de quien forma en investigación, puesto que en algunas ENS quienes han asumido este rol no cuentan con una formación suficiente o una actualización en estos asuntos que les permita acompañar los procesos de una mejor manera. No se desconoce, sin embargo, el esfuerzo que realizan para proveerse de una formación posgradual que les brinde elementos en el quehacer investigativo, pero se requiere que estos conocimientos sean compartidos por todos los maestros que se involucran en este ejercicio. Otro factor es el asunto de los tiempos que deben ser los pertinentes tanto en la malla curricular como en las asignaciones académicas de las maestras y maestros formadores, y los tiempos con que se debe contar para la gestión de conocimiento para que el cuerpo profesoral no se encuentre en la disyuntiva entre enseñar e investigar sino que este sea un proceso articulado que tenga las condiciones necesarias para sacarlo avante.

\section{Conclusiones}

En lo que respecta a la producción de conocimiento a partir de la investigación en las ENS, se encontró que los énfasis más notables en las ENS participantes del estudio, son el pedagógico y didáctico, el ambiental, el desarrollo del pensamiento científico y matemático, las competencias comunicativas y lectoescritoras, la convivencia y ambiente escolar y el de memoria y cultura; esferas temáticas en las cuales se insertan unos procesos de indagación que intentan abordar problemáticas del contexto en el cual se desarrollan las prácticas de los maestros en formación. En cuanto a la formalización de dichos procesos, se observan algunas dificultades en el esbozo de las referencias teóricas y en lo metodológico, lo cual denota una cierta precariedad y falta de rigor y una mirada casi intuitiva sobre el problema que se quiere abordar. No obstante lo anterior, se debe resaltar que todos los ejercicios investigativos llevados a cabo durante el periodo en estudio (2010-2015) demuestran un interés por tratar situaciones de la realidad inmediata que no solo se circunscriben al ámbito escolar, sino que se salen de este marco para interesarse por aquellas cruciales relacionadas con el cuidado del ambiente, con la cultura e identidad.

Sobre las estrategias de las ENS para desarrollar procesos investigativos en la formación de los estudiantes del PFC, puede decirse que, pese a que hay una ruta explícita para dicha formación consistente en el desarrollo de los trabajos de grado desde una fundamentación teórica, un diagnóstico, la generación de un anteproyecto, su aplicación y posterior sistematización y socialización, se requeriría de un mayor énfasis en la fundamentación teórica, en términos de lo epistemológico y lo metodológico, que permitiera superar las falencias identificadas en la revisión documental de los trabajos de grado.

Se consideran problemáticos los tiempos curriculares para la investigación de los maestros en formación (una hora semanal), como los tiempos de los maestros formadores para el acompañamiento de estos procesos, dado que parece que no existe una adecuada relación en términos de horas y estudiantes para asesorar. Es relevante la reflexión pedagógica que los estudiantes en formación desarrollan frente a la práctica investigativa y que presentan en sus trabajos de grado, en donde hacen explícitos sus logros, dificultades y las transformaciones que el desarrollo de sus trabajos ha hecho en la práctica investigativa, como una gran posibilidad de constitución de saber pedagógico que, también, requiere ser sistematizado.

En relación con las estrategias empleadas por las escuelas normales superiores para adelantar los procesos de apropiación social y divulgación del conocimiento que se generan a partir de sus procesos investigativos, son débiles en la totalidad de las instituciones participantes en el proyecto. No cuentan con un sistema que permita desarrollar estos procesos, en tanto, en primer lugar, no existe disponibilidad de maestros para dedicarse a los procesos de sistematización de la información; en segundo lugar, porque no se cuenta con los medios electrónicos para adelantar 
el procesamiento de la información y en tercer lugar porque existe una alta rotación de los maestros que hacen parte del PFC. Estas falencias hacen que los avances en los procesos de investigación se vean afectados cuando los maestros no continúan en las instituciones; así los proyectos tienen más un carácter individual que institucional.

La definición colectiva de conceptualizaciones básicas sobre la investigación y la institucionalización de procesos en las ENS brindarían elementos de base, no solo para la producción y gestión de conocimiento, sino para la generación de una cultura investigativa; esto es, constituir una política interna de investigación y colectivos internos de investigación. A partir de esta política y de los ejercicios que con recursos pertinentes desarrollen los maestros formadores, puede pensarse y vivirse la investigación como una posibilidad de cualificar prácticas, de generar proyectos para la formación posgradual y de asumirse como maestras y maestros investigadores.

\section{Referencias}

Calvo, G., Camargo, M. y Pineda, C. (2008). ¿Investigación educativa o investigación pedagógica? El caso de la investigación en el Distrito Capital. Magis. Revista Internacional de Investigación en Educación, 1(1), 163-173. Recuperado de http://www.redalyc.org/ pdf/2810/281021687011.pdf

Consejo Nacional de Acreditación de Escuelas Normales Superiores (CAENS). (2000). Formación de maestros, elementos para el debate. Bogotá: autor. Recuperado de https://www.mineducacion.gov.co/1621/articles-85742_Archivo_pdf1.pdf
Denzin, N. y Lincoln, Y. (2005). Introduction. The discipline and practice of qualitative research. En N. Denzin y Y. Lincoln, The Sage Handbook of Qualitative Research (3. - ed.) (pp. 1-32). Thousand Oaks: Sage Publications. Recuperado de https://www.sagepub.com/sites/ default/files/upm-binaries/17670_Chapter1.pdf

Farfán, D. y Garzón, M. (2006). La gestión del conocimiento. Documentos de Investigación 29. Bogotá: Editorial Universidad del Rosario. Recuperado de http://repository. urosario.edu.co/bitstream/handle/10336/1207/ BI\%2029.pdf?sequence

Herrera, J. (2010). La formación de docentes investigadores: el estatuto científico de la investigación pedagógica. Magis. Revista Internacional de Investigación en Educación, 5 (3), 53-62. Recuperado de http://www.redalyc. org/articulo.oa?id=281023476003

Ministerio de Educación Nacional (MEN). (2000). Acreditación de calidad y desarrollo de las Escuelas Normales Superiores. Documento marco. Bogotá: autor. Recuperado de https://www.mineducacion.gov.co/cvn/1665/ articles-89942_archivo_pdf.pdf

Ministerio de Educación Nacional (MEN). (2008). Decreto 4790, por el cual se establecen las condiciones básicas de calidad del programa de formación complementaria de las escuelas normales superiores y se dictan otras disposiciones. Bogotá: autor. Recuperado de http://sancionatorios.mineducacion.gov.co/files/Decreto4790.pdf

Ministerio de Educación Nacional (MEN) y Asociación Nacional de Escuelas Normales Superiores (ASONEN). (2015). Naturaleza y retos de las escuelas normales superiores. Bogotá: autor. Recuperado de https:// www.mineducacion.gov.co/1759/articles-345485_ recurso_1.pdf 